\title{
Robust Phase Unwrapping via Deep Image Prior for Quantitative Phase Imaging
}

\author{
Fangshu Yang ${ }^{\circledR}$, Student Member, IEEE, Thanh-An Pham ${ }^{\circledR}$, Graduate Student Member, IEEE, \\ Nathalie Brandenberg ${ }^{\circledR}$, Matthias P. Lütolf, Jianwei Ma ${ }^{\circledR}$, Senior Member, IEEE, \\ and Michael Unser ${ }^{\circledR}$, Fellow, IEEE
}

\begin{abstract}
Quantitative phase imaging (QPI) is an emerging label-free technique that produces images containing morphological and dynamical information without contrast agents. Unfortunately, the phase is wrapped in most imaging system. Phase unwrapping is the computational process that recovers a more informative image. It is particularly challenging with thick and complex samples such as organoids. Recent works that rely on supervised training show that deep learning is a powerful method to unwrap the phase; however, supervised approaches require large and representative datasets which are difficult to obtain for complex biological samples. Inspired by the concept of deep image priors, we propose a deep-learning-based method that does not need any training set. Our framework relies on an untrained convolutional neural network to accurately unwrap the phase while ensuring the consistency of the measurements. We experimentally demonstrate that the proposed method faithfully recovers the phase of complex samples on both real and simulated data. Our work paves the way to reliable phase imaging of thick and complex samples with QPI.
\end{abstract}

Index Terms-Phase unwrapping, deep learning, deep image prior, quantitative phase imaging.

\section{INTRODUCTION}

$\mathbf{I}$ $\mathrm{N}$ RECENT years, three-dimensional stem-cell cultures, called organoids, have emerged as an ideal ex vivo model in regenerative medicine, disease modeling, and studies of biological tissues [1], [2]. For such samples, the privileged imaging modalities are fluorescence-based techniques [1].

Manuscript received October 19, 2020; revised May 19, 2021; accepted July 17, 2021. Date of publication July 30, 2021; date of current version August 10, 2021. This work was supported in part by the National Natural Science Foundation of China under Grant 41625017 and in part by the European Research Council (ERC) through the European Union's Horizon 2020 Research and Innovation Programme under Grant 692726. The associate editor coordinating the review of this manuscript and approving it for publication was Prof. Leyuan Fang. (Corresponding author: Jianwei Ma.)

Fangshu Yang is with the School of Mathematics, Institute of Artificial Intelligence, Harbin Institute of Technology, Harbin 150001, China (e-mail: yfs2016@hit.edu.cn).

Thanh-An Pham and Michael Unser are with the Biomedical Imaging Group, École Polytechnique Fédérale de Lausanne (EPFL), 1015 Lausanne, Switzerland (e-mail: thanh-an.pham@epfl.ch; michael.unser@epfl.ch).

Nathalie Brandenberg and Matthias P. Lütolf are with the Laboratory of Stem Cell Bioengineering, École Polytechnique Fédérale de Lausanne (EPFL), 1015 Lausanne, Switzerland (e-mail: nathalie.brandenberg@epfl.ch; matthias.lutolf@epfl.ch).

Jianwei Ma is with the School of Earth and Space Sciences, Peking University, Beijing 100871, China, and also with the School of Mathematics, Institute of Artificial Intelligence, Harbin Institute of Technology, Harbin 150001, China (e-mail: jwm@pku.edu.cn).

This article has supplementary downloadable material available at https://doi.org/10.1109/TIP.2021.3099956, provided by the authors.

Digital Object Identifier 10.1109/TIP.2021.3099956
Recent works have shown that quantitative phase imaging (QPI) [3] can be used to complement fluorescence-based techniques [4]-[8] or to monitor the rates of growth and morphological changes over an extended period of time [8], [9]. The abundant literature as well as the existence of commercial modules suggest that QPI and multimodal imaging are mature and relevant approaches to study biological samples. In practice, the measured phase suffers from wrapping (i.e., modulo $2 \pi$ of the original phase), which introduces nonrepresentative discontinuities in its distribution. Once recovered from the measurements, the unwrapped version provides quantitative information on the sample [10]. This process, known as phase unwrapping, is an important step for phase imaging. However, its application to biological specimens such as organoids is challenging; in particular, the advent of thick and complex samples calls for advanced methods. Classical methods, largely optimized for the analysis of twodimensional (2D) samples, exhibit important unwrapping artifacts and thus remain challenging to use reliably for these complex samples (see Figure 1). In this work, we propose a method with untrained convolutional neural networks to solve this challenging task.

\section{A. Classical Methods}

In the past decades, numerous $2 \mathrm{D}$ phase-unwrapping algorithms have been proposed. These approaches generally fall into four categories: path following [11], [12], minimum $L_{p}$-norm [13]-[15], Bayesian/regularization [16], [17], and parametric modeling [18].

Most of the path-following algorithms perform a line integration along some path established by techniques such as the branch-cut algorithm [11]. Generally, the path-following methods encounter issues of consistency as the resulting unwrapped phase depends on the path.

By contrast, the minimum-norm methods are global. They estimate the unwrapped phase by minimizing an $L_{p}$-norm. When $p=2$ (least-squares methods) [19], there exist approximate solutions which can be obtained by fast Fourier transforms or discrete cosine transforms [14]. However, the $L_{2}$-norm tends to smooth image edges, especially at the discontinuities [13]. The drawback associated to $p=2$ can be overcome by setting $0 \leq p \leq 1$, which usually increases the computational cost. Bioucas-Dias and Valadao [20] introduced a specific energy-minimization framework for 


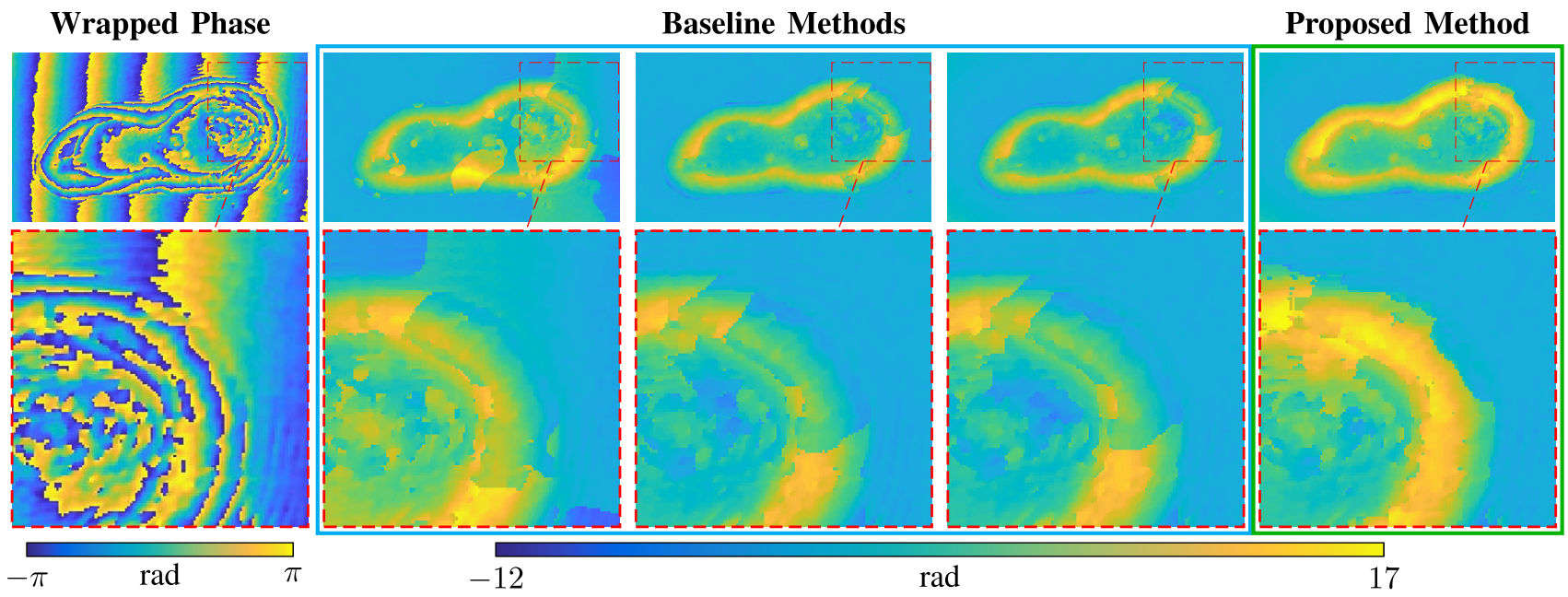

Fig. 1. Example of phase image of organoids. First column: measured (wrapped) phase image. Second to fifth columns: baseline methods (LS, IRTV, and PUMA) and the proposed method (PUDIP). First row: reconstructed phase. Second row: zoomed inset. The size of the unwrapped phase image is $(200 \times 350)$. For the sake of clarity, we removed the non-flat (smooth) background of each unwrapped phase.

phase unwrapping that is solved via graph-cut optimization, the so-called PUMA: phase unwrapping max-flow algorithm. Recent works have extended this method for other imaging modalities [21], [22]. In the same spirit, Condat $e t$ al. recover the wrap-count with a convex relaxation of the original integer-optimization problem [23]. In [24], the authors describe a weighted energy function combined with a Hessian-Schatten-norm regularization [25]. They optimize the minimization problem with an iterative algorithm, named IRTV: isotropic regularization total variation, based on the alternating direction method of multipliers (ADMM) [26].

Bayesian approaches take into account a data-acquisition model and statistical prior knowledge on the phase. Such approaches are usually computationally prohibitive, but an efficient algorithm was proposed in [17] using a series of dynamic-programming procedures connected by the iterated conditional-modes algorithm [27].

The parametric-modeling algorithms constrain the unwrapped phase to a parametric surface, usually a low-order polynomial [18], which makes the unwrapping method computationally efficient. These approaches yield excellent performance only if the parametric model accurately represents the true phase.

Importantly, an assumption considered by most phaseunwrapping approaches is that the absolute value of the unwrapped phase difference between neighboring pixels is less than $\pi$, the so-called Itoh condition [28].

It is worthy to note that there exist alternative methods for quantitative phase-imaging methods that rely on multiple wavelengths or broadband sources [29]-[32]. An imaging system with multiple wavelength sources typically acquires several images so that the wrapping events occur at different locations, thus facilitating the unwrapping task. While our work mainly focuses on a single-wavelength source, our proposed framework can be adapted to the multi-wavelength setting. We refer to the recent reviews on QPI and their detailed description [8], [33].

\section{B. Deep-Learning-Based Approaches}

Recently, deep-learning methods, in particular, convolutional neural networks $(\mathrm{CNN})$, have achieved unprecedented performance in a variety of applications. They surpass conventional methods in diverse fields such as image reconstruction [34], [35], superresolution [36], or x-ray computed tomography [37], and others [38]-[40]. Overall, deep learning in computational imaging is an emerging and promising field of research [41], [42].

To address the 2D phase-unwrapping problem, several works based on deep learning have been proposed. In [43], the authors used a supervised feedforward multilayer perceptron to detect the phase discontinuities in optical Doppler tomography images. More recently, a residual neural network using supervised learning [44] was adopted in [45] to approximate the mapping between the wrapped and the unwrapped phase in the presence of steep gradients. In [46], a CNN-based framework, termed PhaseNet, has been designed. It predicts the wrap-count (integer multiple of $2 \pi$ ) at each pixel, similar to the task of semantic segmentation. Furthermore, a clustering-based postprocessing enforces smoothness by incorporating complementary information. Similar works were proposed in [47], [48]. In [49], the authors improved upon [46] by integrating a network to denoise the noisy wrapped phase. In [50], a generative adversarial network was introduced to effectively suppress the influence of noise. In addition, a framework composed of a residual neural network and the objective function in [24] was proposed in [51] to unwrap quantitative phase images of biological cells.

The aforementioned works rely on supervised learning to learn the mapping between the input-output data pairs. This paradigm needs a large representative training dataset composed of the measured phase and the corresponding ground-truth, which may not be available in many practical applications. In addition, the solutions obtained by direct feedforward networks might be inconsistent with the measurements due to the lack of a feedback mechanism [52]-[54]. 
Nevertheless, these works suggest that $\mathrm{CNN}$ is an appealing solution to the peculiar challenges of phase unwrapping.

\section{Contributions and Roadmap}

In this paper, we introduce a framework with an untrained $\mathrm{CNN}$ for 2D phase unwrapping. Our approach uses the concept of deep image prior (DIP) recently introduced by Ulyanov et al. [55]. We incorporate an explicit feedback mechanism and do not require prior training of the neural network. Taking advantage of these features, we propose a robust and versatile method for phase unwrapping with deep image prior (PUDIP).

The original formulation of phase unwrapping is a nonconvex integer-optimization problem, which contrasts with the usual restoration problems shown in recent works that take advantage of DIP (DIP) [55], [56]. In this work, we show that DIP is also suitable for phase unwrapping, a difficult illposed inverse problem. To the best of our knowledge, it is the first time that DIP is combined with an adaptive loss, which makes our method rather a sequence of DIPs. Not only does this approach improve the reconstructions, but also avoid the destabilization (i.e., significant loss increase and blurred image) that was reported in [55].

In Section II, we introduce the physical model and formulate the computational problem in a variational framework. In Section III, we describe the proposed scheme with an untrained deep neural network. In Section IV, we compare the proposed method against other state-of-the-art (e.g., IRTV, PUMA) approaches on experimental data of organoids. In Section V, we quantitatively assess PUDIP on several simulated data with diverse configurations. We extensively compare our framework with other methods such as the recent deep-learning-based PhaseNet method. The results show that PUDIP improves upon other approaches by taking advantage of model-based and deep-learning worlds. Our work shows that QPI can be applied to large and complex threedimensional samples with higher reliability.

\section{Problem Formulation}

In this section, we formulate the problem of phase unwrapping in a variational framework. Let the region of interest $\Omega \subset \mathbb{R}^{2}$ be discretized into $N$ pixels. To represent the phase of our specimen, we consider the observation model

$$
\boldsymbol{\Phi}=\boldsymbol{\Psi}+2 \pi \mathbf{k},
$$

where $\boldsymbol{\Phi}=\left(\phi_{n}\right) \in \mathbb{R}^{N}$ and $\boldsymbol{\Psi}=\left(\psi_{n}\right) \in[-\pi, \pi)^{N}$ denote the vectorized unwrapped and wrapped phase images, respectively; $\mathbf{k} \in \mathbb{Z}^{N}$ represents the integer multiple of $2 \pi$ referred to as "wrap-count" to be added to the wrapped phase to recover the unwrapped phase. The wrapping process is represented by a function $\mathcal{W}$ applied on the $n$th component of (1) as

$$
\psi_{n}=\mathcal{W}\left(\phi_{n}\right)=\left(\left(\phi_{n}+\pi\right) \bmod (2 \pi)\right)-\pi \in[-\pi, \pi) .
$$
by

The discrete gradient operator $\nabla: \mathbb{R}^{N} \mapsto \mathbb{R}^{N \times 2}$ is given

$$
\nabla \Phi=\left[\begin{array}{ll}
\nabla_{\mathrm{x}} \Phi & \nabla_{\mathrm{y}} \Phi
\end{array}\right]
$$

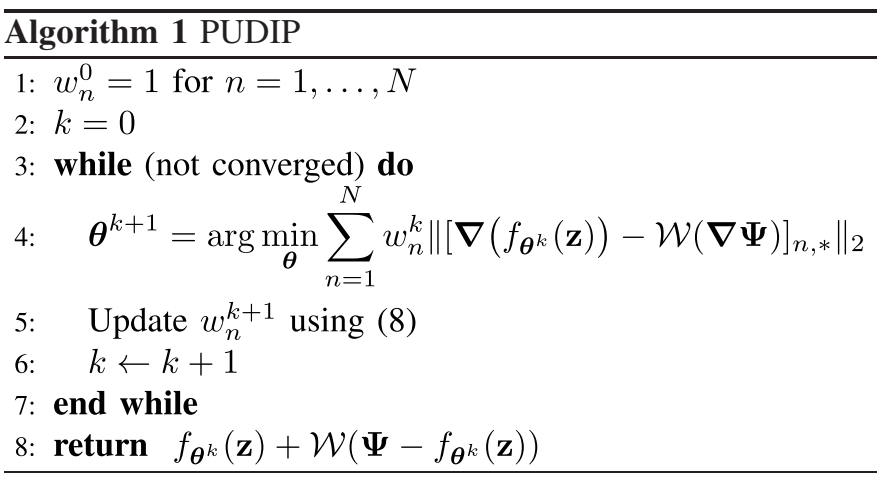

where $\nabla_{\mathrm{x}}: \mathbb{R}^{N} \mapsto \mathbb{R}^{N}$ and $\nabla_{\mathrm{y}}: \mathbb{R}^{N} \mapsto \mathbb{R}^{N}$ denote the horizontal and vertical finite-difference operations, respectively. The phases $\boldsymbol{\Phi}$ and $\boldsymbol{\Psi}$ are related by the equality

$$
\mathcal{W}([\nabla \boldsymbol{\Phi}])=\mathcal{W}([\nabla \boldsymbol{\Psi}]),
$$

where $\mathcal{W}$ is applied component-wise. For 2D phaseunwrapping problems, the phase $\boldsymbol{\Phi}$ satisfies the Itoh continuity condition [28] if

$$
\left\|[\nabla \boldsymbol{\Phi}]_{n, *}\right\|_{2}^{2} \leq \pi^{2}, \quad n \in[1 \ldots N],
$$

where $[\nabla \boldsymbol{\Phi}]_{n, *} \triangleq\left(\left[\nabla_{\mathrm{X}} \boldsymbol{\Phi}\right]_{n},\left[\nabla_{\mathrm{y}} \boldsymbol{\Phi}\right]_{n}\right)$ represents the $n$th component 2D vector of the discrete gradient (i.e., the $n$th row of the matrix $\nabla \boldsymbol{\nabla}$ ). If (5) is satisfied, then (4) simplifies as

$$
[\nabla \boldsymbol{\Phi}]_{n, *}=\mathcal{W}\left([\nabla \boldsymbol{\Psi}]_{n, *}\right), \quad n \in[1 \ldots N] .
$$

Under the hypothesis that a great majority of pixels in $\Phi$ satisfy the constraint condition in (5), we can reconstruct the unwrapped phase by minimizing the weighted energy function [24]

$$
\hat{\boldsymbol{\Phi}}=\arg \min _{\boldsymbol{\Phi} \in \mathbb{R}^{N}} \sum_{n=1}^{N} w_{n}(\boldsymbol{\Phi})\left\|[\nabla \boldsymbol{\Phi}-\mathcal{W}(\boldsymbol{\nabla} \boldsymbol{\Psi})]_{n, *}\right\|_{2},
$$

where $w_{n}(\boldsymbol{\Phi}) \in \mathbb{R}_{\geq 0}$ is the adaptive nonnegative weight for the $n$th component of the cost to relax the restriction. It is defined as

$$
w_{n}(\boldsymbol{\Phi})= \begin{cases}\frac{1}{\left\|[\boldsymbol{\epsilon}]_{n, *}\right\|_{2}}, & \epsilon_{\min } \leq\left\|[\boldsymbol{\epsilon}]_{n, *}\right\|_{2} \leq \epsilon_{\max } \\ \frac{1}{\epsilon_{\max }}, & \left\|[\boldsymbol{\epsilon}]_{n, *}\right\|_{2} \geq \epsilon_{\max } \\ \frac{1}{\epsilon_{\min }}, & \left\|[\boldsymbol{\epsilon}]_{n, *}\right\|_{2} \leq \epsilon_{\min },\end{cases}
$$

where $\epsilon=(\nabla \Phi-\mathcal{W}(\nabla \Psi))$, and where $\epsilon_{\min }$ and $\epsilon_{\max }$ are the user-defined minimum and maximum boundary weights, respectively. Note that (7) can be seen as a shifted isotropic TV and other variants could be of interest for future works [57]. In addition, the solutions can be improved by imposing prior knowledge (i.e., a regularization term) such as a HessianSchatten norm (HS) [25] in an attempt to compensate for the ill-posed nature of the problem.

It is worthy to note that the solution obtained by iteratively minimizing the objective function (7) offers no guarantee regarding the consistency between the rewrapped phase $\mathcal{W}(\hat{\boldsymbol{\Phi}})$ and the wrapped phase $\Psi$ [24]. This is because (7) relies 


\section{Generating Unwrapped Phase}

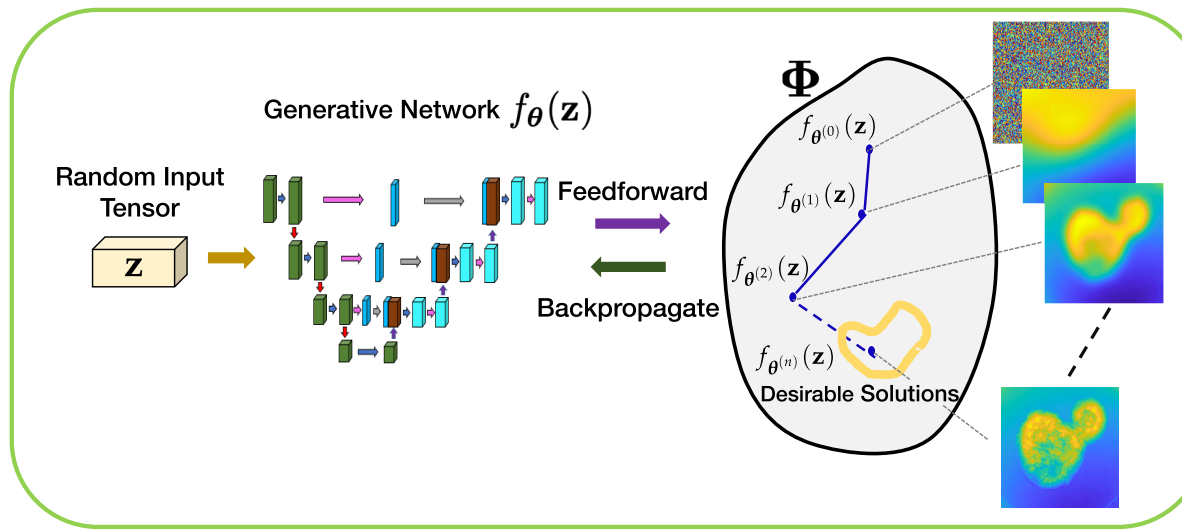

Measurement

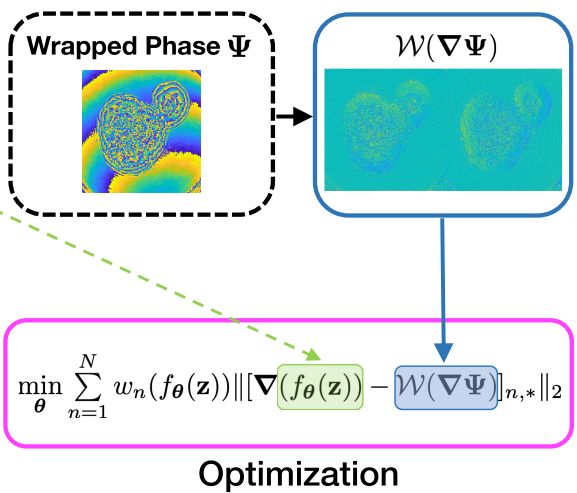
$\mathbf{z} \in \mathbb{R}^{C \times N}:$ Random tensor
$\boldsymbol{\Phi} \in \mathbb{R}^{N} \quad$ : Vectorized unwrapped (true) phase
$\longrightarrow \quad$ : Operation of wrapping and
$f_{\boldsymbol{\theta}}(\mathbf{z}) \in \mathbb{R}^{N}:$ Output of generative network
$\Psi \in \mathbb{R}^{N}$ : Vectorized wrapped phase

Fig. 2. Schematic diagram of the proposed PUDIP, for two-dimensional phase unwrapping. The architecture of the generative network is fully described in the supplementary materials.

on continuous optimization to solve the discrete-optimization problem (1). Therefore, we adopt the single postprocessing step [58]

$$
\tilde{\boldsymbol{\Phi}}=\hat{\boldsymbol{\Phi}}+\mathcal{W}(\boldsymbol{\Psi}-\hat{\boldsymbol{\Phi}}),
$$

where $\tilde{\boldsymbol{\Phi}}$ is the final solution, congruent with the measurement $\boldsymbol{\Psi}$.

\section{Phase Unwrapping With Deep Image Prior}

DIP is a scheme recently introduced in [55]. Rather than learning the mapping between input and output with a large training dataset, DIP handles the inverse problem by assuming that the unknown image can be represented well by the output of an untrained generative network. Recent works have shown the effectiveness of DIP for computational imaging [56], [59]-[61]. In the spirit of this approach, we propose a framework where we restore the unwrapped phase based on this implicit prior.

The unwrapped phase is generated by the CNN given by

$$
\boldsymbol{\Phi}=f_{\boldsymbol{\theta}}(\mathbf{z}),
$$

where $f$ denotes the neural network and $\boldsymbol{\theta}$ stands for the network parameters to be learned. The fixed randomly-initialized vector $\mathbf{z} \in \mathbb{R}^{C \times N}$ acts as input to the generative network, while $C$ is the number of input channels.

Plugging (10) in (7) leads to the optimization problem

$$
\hat{\boldsymbol{\theta}}=\arg \min _{\boldsymbol{\theta}} \sum_{n=1}^{N} w_{n}\left(f_{\boldsymbol{\theta}}(\mathbf{z})\right)\left\|\left[\nabla\left(f_{\boldsymbol{\theta}}(\mathbf{z})\right)-\mathcal{W}(\boldsymbol{\nabla} \boldsymbol{\Psi})\right]_{n, *}\right\|_{2} .
$$

In our optimization approach, we aim at minimizing this loss function by taking advantage of the family of stochastic gradient-descent methods. The schematic diagram of PUDIP is shown in Figure 2.

Finally, we achieve congruence with the single step

$$
\tilde{\boldsymbol{\Phi}}=f_{\hat{\boldsymbol{\theta}}}(\mathbf{z})+\mathcal{W}\left(\boldsymbol{\Psi}-f_{\hat{\boldsymbol{\theta}}}(\mathbf{z})\right) .
$$

The process is described in Algorithm 1, where one can see that PUDIP consists in a sequence of minimization problems.

\section{A. Architecture}

We design a CNN based on the U-Net-like encoder-decoder architecture [55], [62]. The setup includes skip connections with convolution and concatenation. This enables the network to reconstruct the feature maps with both local details and global texture. We set a constant number of channels (i.e., 128) in all the convolutional layers, except for those included in the skip connection whose channel number is 4 . We chose the parametric rectified linear unit [63] as the nonlinear activation function. Furthermore, the downsampling operation is implemented by convolutional modules with strides of 2 , so that the size of the feature map is halved in the contracting path. The upsampling operation doubles the size through bilinear interpolation. The scaling-expanding structure makes the effective receptive field increase at deeper layers [62]. As last stage, we have set one layer that subtracts a scalar value from the image. This scalar takes care of the bias intrinsic to phase unwrapping, which can recover phase only up to a constant. For simulated data, we subtracted the minimum value of the entire image to enforce nonnegativity. For real data, we subtracted the mean value of a top-left area whose dimension is $(30 \times 30)$ and corresponds to a background region (see supplementary materials for detailed architecture).

\section{EXPERIMENTS}

Thick and complex samples present complicated wrapping events and potentially contain a few sharp edges at which the Itoh condition may not hold in the true phase. These combined factors increase the difficulty to unwrap their phase. To illustrate these challenges, we acquired images of organoids with digital holography microscopy and unwrapped their phase using the proposed method as well as other baseline methods. The quality of unwrapped images will impact the subsequent 
steps of image analysis. Hence, we additionally illustrate how segmentation — a typical image processing for QPI [64]—can be altered by the outcome of phase unwrapping.

\section{A. Optimization Strategy}

In our experiments, we adopt the following strategy: The input variable $\mathbf{z}$ is a random vector filled with the uniform noise $U(0,0.1)$. To avoid undetermined gradients with respect to $\boldsymbol{\theta}$ in (11), we offset the norm there by the small constant $\delta=10^{-18}$. In practice, the adaptive weights $w_{n}$ are updated every $N_{w}$ iterations to enforce sparsity in the loss function (11) [24]. We optimize (11) by using the adaptive moment-estimation algorithm (Adam, $\beta_{1}=0.9$ and $\beta_{2}=$ $0.999)$ [65]. The optimization is performed on a desktop workstation (Nvidia Titan X GPU, Ubuntu operating system) and implemented on PyTorch [66]. In our experiments, the random initialization of the input variable did significantly impact neither the performance, nor the time of computation.

\section{B. Parameter Setting}

We set the maximum number of iterations as 2000 (see Supplementary Materials). The hyperparameters of the network were initialized to default values by PyTorch. We used a learning rate of 0.01 . The weights $w_{n}$ were updated every $N_{w}=100$ iterations with $\left[\epsilon_{\min }, \epsilon_{\max }\right]=[0.1,8]$. During a typical optimization, the weights $w_{n}$ will be large in the area around sharp edges [24]. The parameter $\epsilon_{\min }$ prevents that the weights from becoming too large in the early iterations of the global optimization, which would force the corresponding pixels to be irreversibly set to zero. Similarly, $\epsilon_{\max }$ ensures that the weights do not become too small.

To optimize over the synthetic samples, we updated the weights $w_{n}$ with $\left[\epsilon_{\min }, \epsilon_{\max }\right]$. We tried $\left[\epsilon_{\min }, \epsilon_{\max }\right]=[0.1,10]$, $[0.05,20],[0.02,50]$, and $[0.01,100]$, choosing the best performance. The weights were updated every $N_{w}=200$ for the first sample, 100 otherwise. As we randomly initialized the parameters of the network $\boldsymbol{\theta}$, we repeated each experiment five times and report the average performance.

\section{Experimental Setup}

Mouse organoids of the small intestine were released from Matrigel ${ }^{\circledR}$ (Corning) and dissociated into single cells. After centrifugation, the cells were re-suspended at the appropriate density in ENR-CV medium supplemented with Thiazovivin (ReproCell) and seeded to deposit about 100 cells per microwell onto imaging bottom Gri3D hydrogel microwell array plates (SUN bioscience) of 300 micrometer in diameter. The cells were then let to sediment for 30 minutes as such and $150 \mu \mathrm{L}$ of self-renewal medium supplemented with 2\% Matrigel. The stem cells were expanded in selfrenewal for 3 days, and the organoids were differentiated for another 3 days in differentiation medium (ENR) [67]. Once the stem cells underwent morphogenesis and formed fully matured organoids, the organoids were imaged using a digital holographic microscope (T1000-Fluo, LynceeTec). The holograms, phases, and amplitudes were acquired for downstream reconstruction with a pixel of physical length
TABLE I

Baseline Methods. CNN ${ }^{1}$ Denotes the Supervised-Learning Method, While CNN ${ }^{2}$ DenOtes Our Method WITH UNTRAINED NETWORK

\begin{tabular}{|c|c|c|c|}
\hline Method & Reference & Regularization & Optimization \\
\hline GA & [11] & - & branch-cut \\
\hline LS & [14] & - & least-squares \\
\hline IRTV & [24] & HS [25] & ADMM [26] \\
\hline PUMA & [20] & - & graph cut \\
\hline PhaseNet & [46] & - & $\mathrm{CNN}^{1}$ \\
\hline PUDIP & & - & $\mathrm{CNN}^{2}$ \\
\hline
\end{tabular}

of $6.45 \mu \mathrm{m}(\mathrm{NA}=0.3$, magnification $10 \times$, and wavelength $684.6 \mathrm{~nm}$ ). The time interval between each frame was 1 minute for the time-lapse measurements.

\section{Baseline Methods}

We compare the proposed method with other state-of-theart conventional or CNN-based methods such as Goldstein's algorithm (GA) [11], unweighted least-squares algorithm (LS) [14], IRTV ${ }^{1}$ [24], PUMA $^{2}$ [20], and PhaseNet [46] (see Table I). Goldstein's algorithm is a path-following method that adopts the branch-cut strategy based on the phase residues and needs the knowledge of a phase-reference point. By contrast, the LS, IRTV, and PUMA approaches aim at minimizing an objective function and belong to the minimumnorm category. Note that the original LS method, which relies on a continuous optimization, may result in an inconsistent solution, while GA, IRTV, and PUMA always return consistent solutions. To enforce measurement consistency for LS, we adopted the strategy defined by (9). We also compare PUDIP to the recently proposed PhaseNet [46]. We adopted the strategy of [48] to generate a training dataset in two steps. First, the elements of a square matrix whose size varies between $(3 \times 3)$ and $(11 \times 11)$ were randomly generated following a uniform distribution $U(0,1)$ for half of the samples and a Gaussian distribution $\mathcal{N}(0,1)$ followed by the subtraction of the minimum of the matrix for the other half. Then, we multiplied the matrix by a scalar randomly generated following a uniform distribution $U(3 \pi, 12 \pi)$ and upsampled the matrix to a $(256 \times 256)$ image using bicubic interpolation [68]. The obtained data had a maximum value ranging from $2 \pi$ to $40 \pi$. In addition, we only kept the central disk of the generated phase images and filled the background with 0 . The training dataset is composed of 9,600 samples; the size of each image is $(256 \times 256)$. The wrap-count in the training data varies between 0 and 20 , which makes it a 21-class problem (see the details in the supplementary materials). We set the other hyperparameters as in [46] and trained PhaseNet with this generated dataset for all the experiments.

\footnotetext{
${ }^{1}$ The source code for IRTV is available from https://cigroup.wustl.edu/ publications/open-source/

${ }^{2}$ The source code for PUMA is available from http://www.lx.it.pt/ $\sim$ bioucas/code.htm
} 
All model-based methods were run on a desktop computer (Intel XeonE5-1650 CPU, 3.5 GHz, $32 \mathrm{~GB}$ of RAM) and implemented in MATLAB R2019a. For their implementation, we initialized the unwrapped phase with $\mathbf{0} \in \mathbb{R}^{N}$. All parameters were set and optimized according to the guidelines provided by the authors. Specifically, the regularization parameter for the Hessian-Schatten-norm regularization in IRTV was set between $10^{-3}$ and $10^{-1}$. In PUMA, we set the non-convex quantized potential of exponent $p=0.5$, the quadratic region threshold as 0.5 , and the high-order cliques $[1,0],[0,1],[1,1]$, and $[-1,1]$.

PUDIP takes about 100 seconds on GPU to unwrap a $(256 \times 256)$ image with 1000 iterations. In comparison, PUMA and IRTV take about 2 and 380 seconds on CPU, respectively.

\section{E. Post-Processing of the Unwrapped Phase}

The microwells in which the organoids are loaded induce a non-flat (smooth) background. For the sake of clarity, we removed the background of each unwrapped phase. We estimated the background by fitting a polynomial of degree 3 in background areas. To detect the background, we applied a $(3 \times 3)$ standard-deviation filter on the unwrapped image. We defined the background as any pixel below a certain threshold $T_{\sigma} \in[0.5,1]$.

\section{F. Phase Unwrapping of Organoids}

The results of various methods are shown in Figure 3. The LS method yields inaccurate results over large areas, such as non-flat background or disrupted structures. In comparison, the three other approaches perform better. However, some areas pointed out by the rectangle exhibit sudden breaks in the phase unwrapped by IRTV and PUMA. The phase is expected to be relatively smooth since the epithelium of the organoids consists in a continuous layer of cells, forming then the border of the sample [69]. By contrast, PUDIP better recovers it for all samples.

PhaseNet failed to reconstruct the unwrapped phase in all cases (see the supplementary materials), most probably because the training set is not adequate for our experimental data. Likewise, GA was unable to recover the samples. The solutions found by PhaseNet and GA exhibit several areas with values higher than their surrounding, which does not accurately represent the characteristic features found in intestinal organoids, such as the epithelium and the lumen.

In the first row of Figure 3, the unwrapped phase might deviate from the phase image predicted by the straightray approximation [70] in the center part where it is nonsmooth. The approximation is accurate if the wavelength is much smaller than the features of the sample (e.g., local inhomogeneity of the refractive index). The mismatches are then likely to occur in the areas where the features are, which suggests that local inhomogeneities are present in the inner part.

In addition, we computed the relative error between the rewrapped phase $\mathcal{W}(\tilde{\boldsymbol{\Phi}})$ and the wrapped phase $\boldsymbol{\Psi}$ defined as

$$
\Delta \epsilon(\mathcal{W}(\tilde{\boldsymbol{\Phi}}), \boldsymbol{\Psi})=\frac{\|\boldsymbol{\Psi}-\mathcal{W}(\tilde{\boldsymbol{\Phi}})\|_{2}}{\|\boldsymbol{\Psi}\|_{2}} .
$$

The relative errors of all methods are lower than $10^{-13}$, which indicates that the results are congruent with the measurements up to rounding errors.

\section{G. Phase Unwrapping of Time-Lapse Measurements}

Further, we acquired time-lapse measurements of organoids to validate the benefits of our approach in sequential imaging. In the last frames, the size of the organoids increases and the intra-organoid composition becomes visibly more heterogeneous. It is noteworthy that the intestinal organoids are absorbing water as they grow over time [71], which explains that the phase value gets closer to the background value. Because of more complex wrapping events, the unwrapping task becomes even more challenging. By using PUDIP, we show here that the borders as well as the flatness of the background are well preserved (see Figure 4). On the contrary, the unwrapped phase of the other methods either result in a background with unlikely $2 \pi$ jumps or borders with sudden breaks.

\section{H. Segmentation of Time-Lapse Measurements}

Image segmentation is a step that one would usually perform on the unwrapped phase [64]. Our aim now is to illustrate how unwrapping can affect the segmentation results. To that end, we simply thresholded the images obtained from the different methods with a threshold set at $20 \%$ of the maximal value.

In Figure 5, we observe that the segmentation is especially impacted at the borders where sudden breaks occur in the unwrapped phase. In all frames, the segmentation of PUDIP solutions preserves the integrity of the boundaries better than the other methods.

\section{Simulated Data}

While the results on experimental data are encouraging, we want to quantitatively assess the quality of our proposed method. To that end, we simulated the acquisition of phase images of organoid-like samples. In addition, we generated diverse artificial data which are similar to those found in [20] and [48] (see the details in the supplementary materials).

\section{A. Quantitative Evaluation}

We quantitatively evaluate the quality of the reconstructed phase $\tilde{\boldsymbol{\Phi}}$ with respect to the ground-truth $\boldsymbol{\Phi}$. Our first metric is the regressed signal-to-noise ratio (RSNR) defined as

$$
\operatorname{RSNR}(\tilde{\boldsymbol{\Phi}}, \boldsymbol{\Phi})=\max _{b \in \mathbb{R}^{+}}\left(20 \log _{10}\left(\frac{\|\boldsymbol{\Phi}\|_{2}}{\|(\tilde{\boldsymbol{\Phi}}+b)-\boldsymbol{\Phi}\|_{2}}\right)\right),
$$

where $\|\cdot\|_{2}$ denotes the $L_{2}$ norm and where $b$ adjusts for a potential global offset. This adjustment is used in the interest of fairness, because phase unwrapping can only recover the phase up to a constant. When the RSNR is more than $100 \mathrm{~dB}$, the recovered phase image differs from the ground truth because of numerical imprecision and not because of wrong 

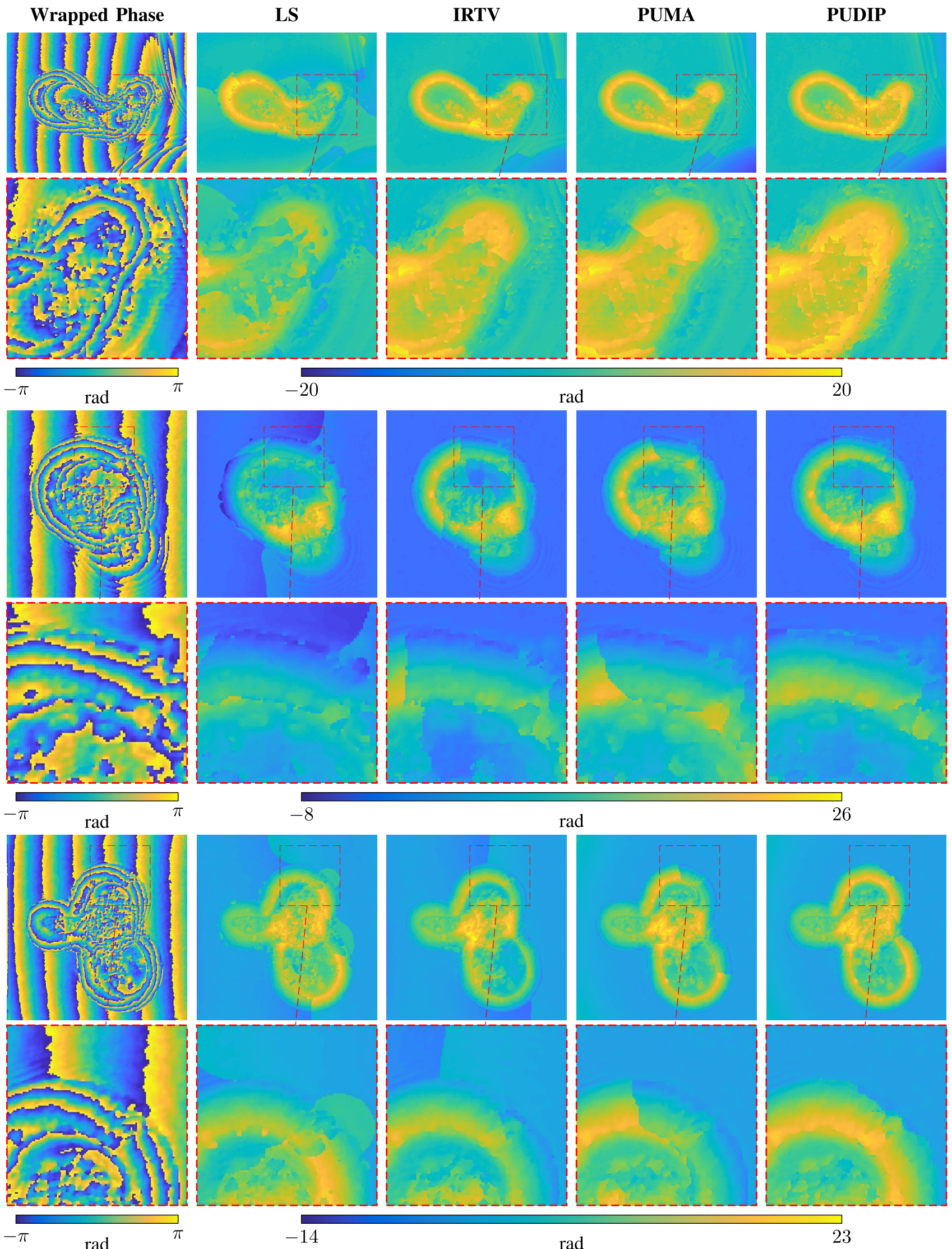

$\operatorname{rad}$

26

Fig. 3. Reconstructed phase images of organoids. First column: measured (wrapped) phase image. Second to fifth columns: algorithms using LS, IRTV, PUMA, and the proposed method (PUDIP). First row: reconstructed phase. Second row: zoomed inset. The size of the unwrapped phase image is (350 $\times 450$ ), $(260 \times 250)$, and $(360 \times 350)$, respectively. For the sake of clarity, we removed the non-flat (smooth) background of each unwrapped phase. 


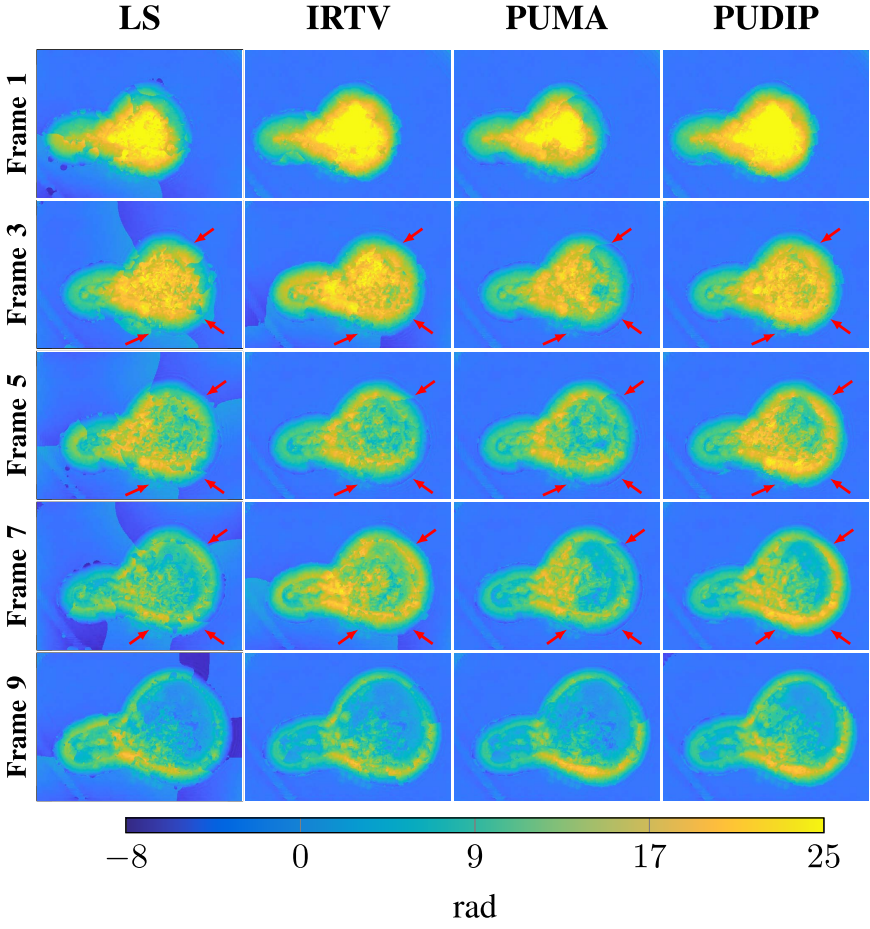

Fig. 4. Time-lapse reconstructions. The images were saturated for visualization purpose. The size of the unwrapped phase image is $(280 \times 390)$. For the sake of clarity, we removed the non-flat (smooth) background of each unwrapped phase.

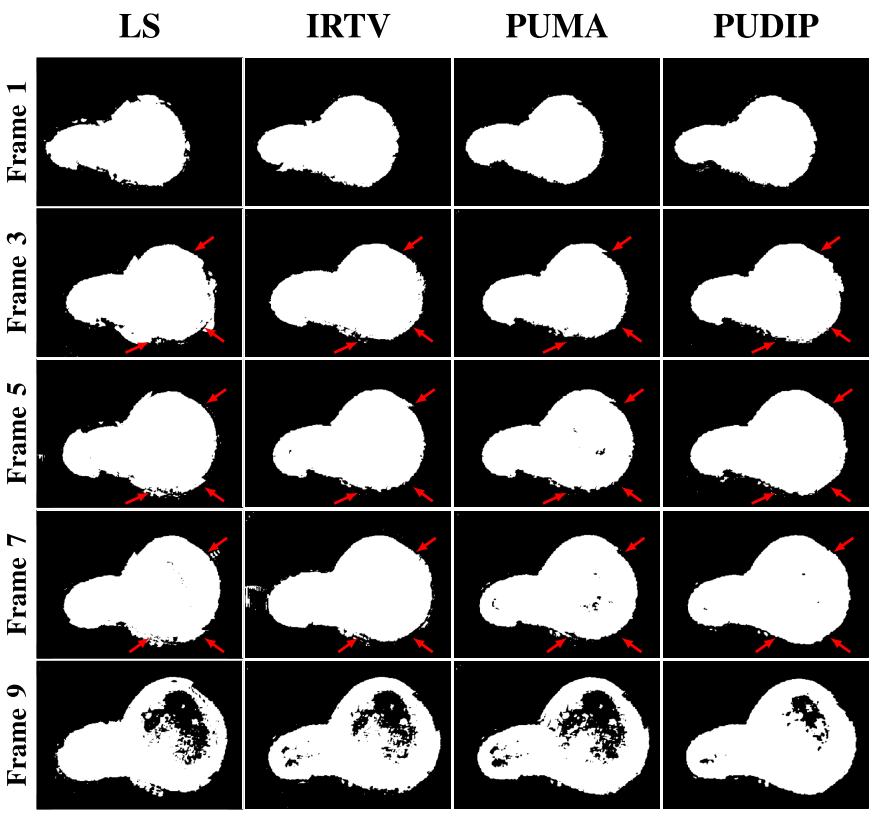

Fig. 5. Segmentation of time-lapse reconstructions. We thresholded at $20 \%$ of the maximum value of the image.

unwrapping. We therefore set the corresponding value to infinity. In addition, we compute the structural similarity (SSIM) defined as

$$
\operatorname{SSIM}(\tilde{\boldsymbol{\Phi}}, \boldsymbol{\Phi})=\frac{\left(2 \mu_{\boldsymbol{\Phi}} \mu_{\tilde{\boldsymbol{\Phi}}}+c_{1}\right)\left(2 \sigma_{\boldsymbol{\Phi}} \sigma_{\tilde{\boldsymbol{\Phi}}}+c_{2}\right)}{\left(\mu_{\boldsymbol{\Phi}}^{2}+\mu_{\tilde{\boldsymbol{\Phi}}}^{2}+c_{1}\right)\left(\sigma_{\boldsymbol{\Phi}}^{2}+\sigma_{\tilde{\boldsymbol{\Phi}}}^{2}+c_{2}\right)}
$$

TABLE II

RSNR [dB] AND SSIM OF THE RECONSTRUCTED-Phase IMAgES Versus THE ANGLE of CRopPING. THE RSNR AND SSIM OF OUR METHOD (PUDIP) ARE THE AVERAGE OF FIVE EXPERIMENTS

\begin{tabular}{|c|c|c|c|c|c|c|c|}
\hline & | Angle & GA & LS & IRTV & PUMA & PhaseNet & PUDIP \\
\hline \multirow{7}{*}{ 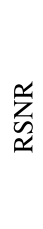 } & $0^{\circ}$ & $\infty$ & $\infty$ & $\infty$ & $\infty$ & 24.79 & $\infty$ \\
\hline & $45^{\circ}$ & 6.80 & 5.15 & 8.61 & 10.20 & 14.10 & 15.99 \\
\hline & $90^{\circ}$ & 2.70 & 2.86 & 3.15 & 3.87 & 22.14 & 37.75 \\
\hline & $135^{\circ}$ & -0.56 & 1.32 & 2.46 & 2.06 & 22.01 & 43.52 \\
\hline & $180^{\circ}$ & -5.15 & -0.13 & 0.84 & $\infty$ & 19.33 & $\infty$ \\
\hline & $225^{\circ}$ & -6.70 & -0.43 & -0.24 & 2.21 & 19.96 & 41.44 \\
\hline & $270^{\circ}$ & -8.00 & -1.85 & -1.66 & 2.01 & 21.23 & $\infty$ \\
\hline \multirow{7}{*}{ 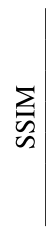 } & $0^{\circ}$ & 1.0000 & 1.0000 & 1.0000 & 1.0000 & 0.9799 & 1.0000 \\
\hline & $45^{\circ}$ & 0.8975 & 0.8346 & 0.9429 & 0.9595 & 0.9680 & 0.9866 \\
\hline & $90^{\circ}$ & 0.9074 & 0.7180 & 0.7337 & 0.7418 & 0.9772 & 0.9995 \\
\hline & $135^{\circ}$ & 0.8360 & 0.5716 & 0.6510 & 0.5576 & 0.9769 & 1.0000 \\
\hline & $180^{\circ}$ & 0.4863 & 0.4772 & 0.4893 & 1.0000 & 0.9771 & 1.0000 \\
\hline & $225^{\circ}$ & 0.4269 & 0.3411 & 0.3225 & 0.1183 & 0.9858 & 1.0000 \\
\hline & $270^{\circ}$ & 0.3655 & 0.2395 & 0.2246 & 0.0838 & 0.9907 & 1.0000 \\
\hline
\end{tabular}

TABLE III

RSNR [dB] AND SSIM OF THE RECONSTRUCTED-PHASE IMAGES VERSUS the MaXimal Value. The RSNR and SSIM of OUR Method (PUDIP) ARE THE AVERAGE OF FIVE ExPERIMENTS

\begin{tabular}{|c|c|c|c|c|c|c|c|}
\hline & Max value & GA & LS & IRTV & PUMA & PhaseNet & PUDIP \\
\hline \multirow{7}{*}{ 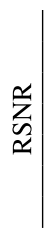 } & 6 & 5.69 & 13.12 & $\infty$ & $\infty$ & -5.71 & $\infty$ \\
\hline & 12 & 1.02 & -0.39 & 11.31 & $\infty$ & 1.25 & $\infty$ \\
\hline & 18 & 1.45 & 1.20 & 3.22 & $\infty$ & 5.62 & $\infty$ \\
\hline & 24 & 3.85 & 0.21 & 4.99 & 5.69 & 8.95 & $\mathbf{7 8 . 5 4}$ \\
\hline & 30 & 5.20 & 1.04 & 7.38 & 7.62 & 8.35 & 28.53 \\
\hline & 36 & 4.62 & 0.48 & 8.71 & 9.18 & 10.13 & 25.70 \\
\hline & 42 & 14.53 & 0.94 & 4.37 & 10.52 & 12.46 & 27.74 \\
\hline \multirow{7}{*}{ 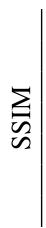 } & 6 & 0.9299 & 0.9834 & 1.0000 & 1.0000 & 0.7105 & 1.0000 \\
\hline & 12 & 0.9258 & 0.5989 & 0.3616 & 1.0000 & 0.7788 & 1.0000 \\
\hline & 18 & 0.9311 & 0.5539 & 0.6481 & 1.0000 & 0.8067 & 1.0000 \\
\hline & 24 & 0.9453 & 0.5312 & 0.6411 & 0.5873 & 0.8298 & 0.9990 \\
\hline & 30 & 0.9551 & 0.5160 & 0.6435 & 0.5866 & 0.8168 & 0.9977 \\
\hline & 36 & 0.9532 & 0.5044 & 0.6416 & 0.5796 & 0.8224 & 0.9957 \\
\hline & 42 & 0.9782 & 0.4951 & 0.6364 & 0.5784 & 0.8597 & 0.9959 \\
\hline
\end{tabular}

where $\mu_{\Phi}, \mu_{\tilde{\Phi}}, \sigma_{\Phi}, \sigma_{\tilde{\Phi}}$, and $\sigma_{\Phi \tilde{\Phi}}$ are the local means, standard deviations, and cross-covariance for images $\boldsymbol{\Phi}, \tilde{\boldsymbol{\Phi}}$, respectively. The regularization constants $c_{1}=10^{-4}$ and $c_{2}=9 \times 10^{-4}$ avoid instabilities over image regions where the local mean or standard deviation is vanishing.

\section{B. Simulated Phase Images of Organoid-Like Sample}

In order to obtain a physically-realistic ground-truth, we simulated the wave propagation through the sample with the beam-propagation method [72]. From the threedimensional simulation, we directly obtain the wrapped phase. Under the straight-ray approximation [70], we expect that the unwrapped phase is proportional to the integral of the refractive index differences. We therefore refer to the straight-ray approximation $\boldsymbol{\Phi}_{\text {sr }}$ as the ground-truth. As shown in Figure 6, the phase unwrapped by PUDIP is consistent with $\boldsymbol{\Phi}_{\mathrm{sr}}$. The solutions of the other methods have wrongly unwrapped areas. The entanglement of several elements complicates the wrapping events in those areas (Figure 6 top right panel). The fact that some parts are defocused adds to the 

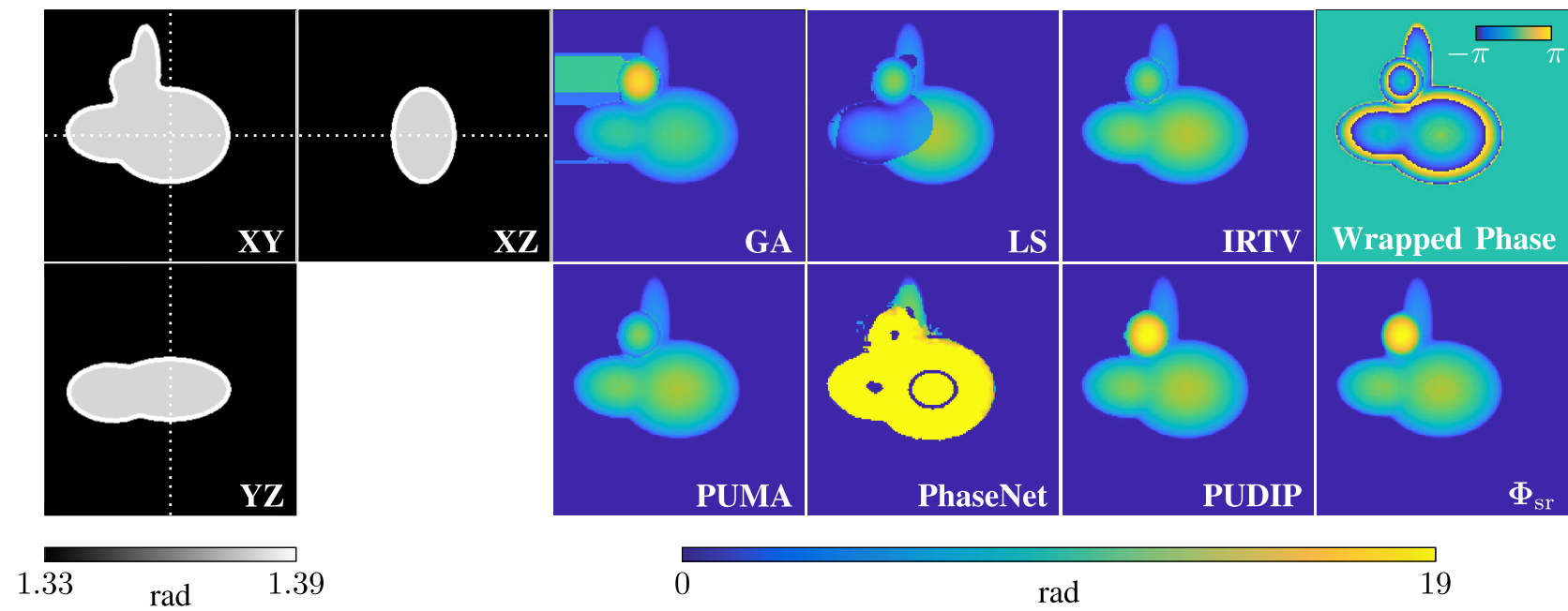

$$
0
$$

$\mathrm{rad}$

19

Fig. 6. Organoid-like reconstructions. The images were saturated for visualization purpose. The size of the unwrapped phase image is $(159 \times 159)$. The first two columns are orthographic slices of the three-dimensional (3D) distribution of refractive indices. All slices include the center of the volume. From the third to fifth column, the text gives the method used to unwrap. The wrapped phase resulting from $3 \mathrm{D}$ simulation and the ground-truth $\boldsymbol{\Phi}_{\text {sr }}$ are displayed in the last column (from top to bottom).
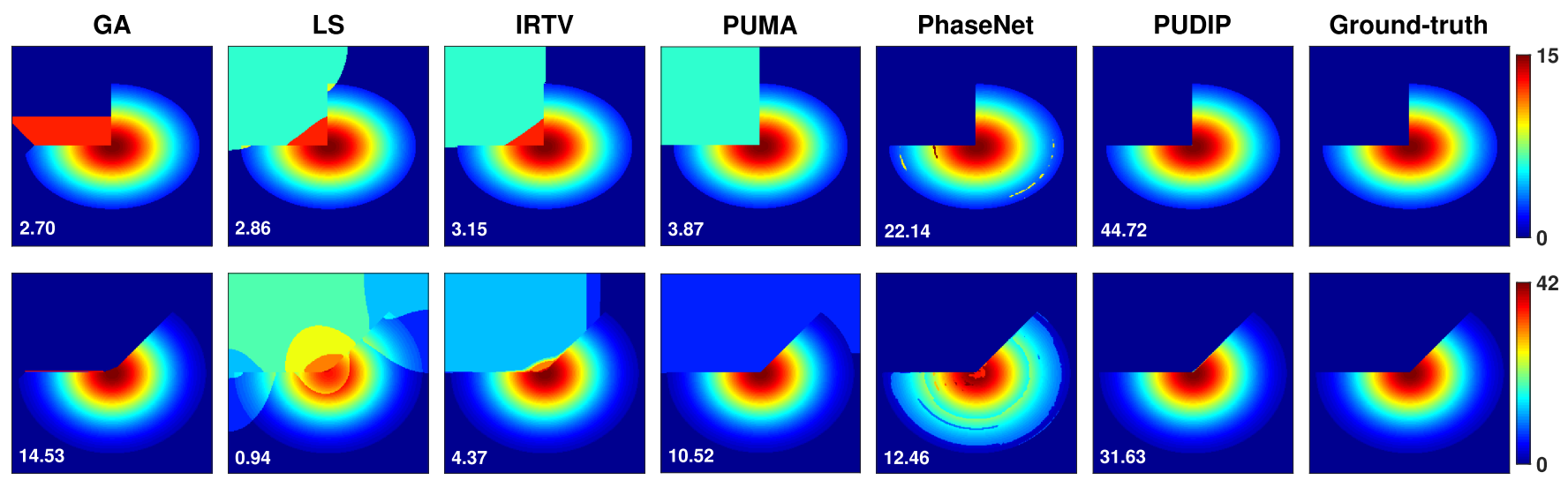

Fig. 7. Unwrapped phases of two simulated samples. From left to right, the results are obtained by GA, LS, IRTV, PUMA, PhaseNet, and our approach (PUDIP). The ground-truth images are presented in the last column. The corresponding RSNR [dB] is showed at the left bottom of each subfigure.

challenge since ripples are present around the border. The slightly defocused parts are wrongly estimated by baseline methods, which impacts the whole unwrapping result. It is worthy to note that real data also have ripples around the border, which might partially explain the difficulty to unwrap phase images of organoids. We provide more examples in the supplementary materials.

\section{Phase Unwrapping of Artificial Images}

We generated three kinds of samples similar to previous works [20], [48]. The first and second categories consist of ellipses. In the first type of sample, we cropped the ellipses with angles ranging from $0^{\circ}$ to $270^{\circ}$ with an increment of $45^{\circ}$. In the second type of sample, we scaled the phase image (i.e., an ellipse cropped with a fixed angle) so that its maximum was in the range of 6 to 42 with an increment of 6 . The last kind of sample is the same as the one we used to train PhaseNet (see Section IV-D). We use these images to test our method on samples usually seen in other modalities [20].
When the unwrapping task is relatively simple, all the baseline methods, as well as our method, perform well (see the first row in Tables II and III). When the phase images are more complex (e.g., when a few pixels violate the Itoh condition), all the conventional methods lead to blocky errors. As expected, PhaseNet wrongly estimates the unwrapped phases when they differ from the training set. On the contrary, our framework with untrained CNN faithfully unwraps the phase for nearly all configurations (Tables II and III). In Figure 7, one can observe some typical unwrapping behavior of the different methods, as well as the obtained RNSR.

For the last type of samples, deep learning techniques perform better than the conventional techniques (Table IV). Since the training and testing sets match, PhaseNet is quantitatively more accurate than PUDIP. As reported in [55], supervised schemes tend to outperform unsupervised approaches when the training and testing sets are consistent. It is noteworthy that PUDIP commits errors only at the border of the disk and that the large discrepancy in the RSNR between PhaseNet and PUDIP mainly comes from the fact that any error is likely to be 

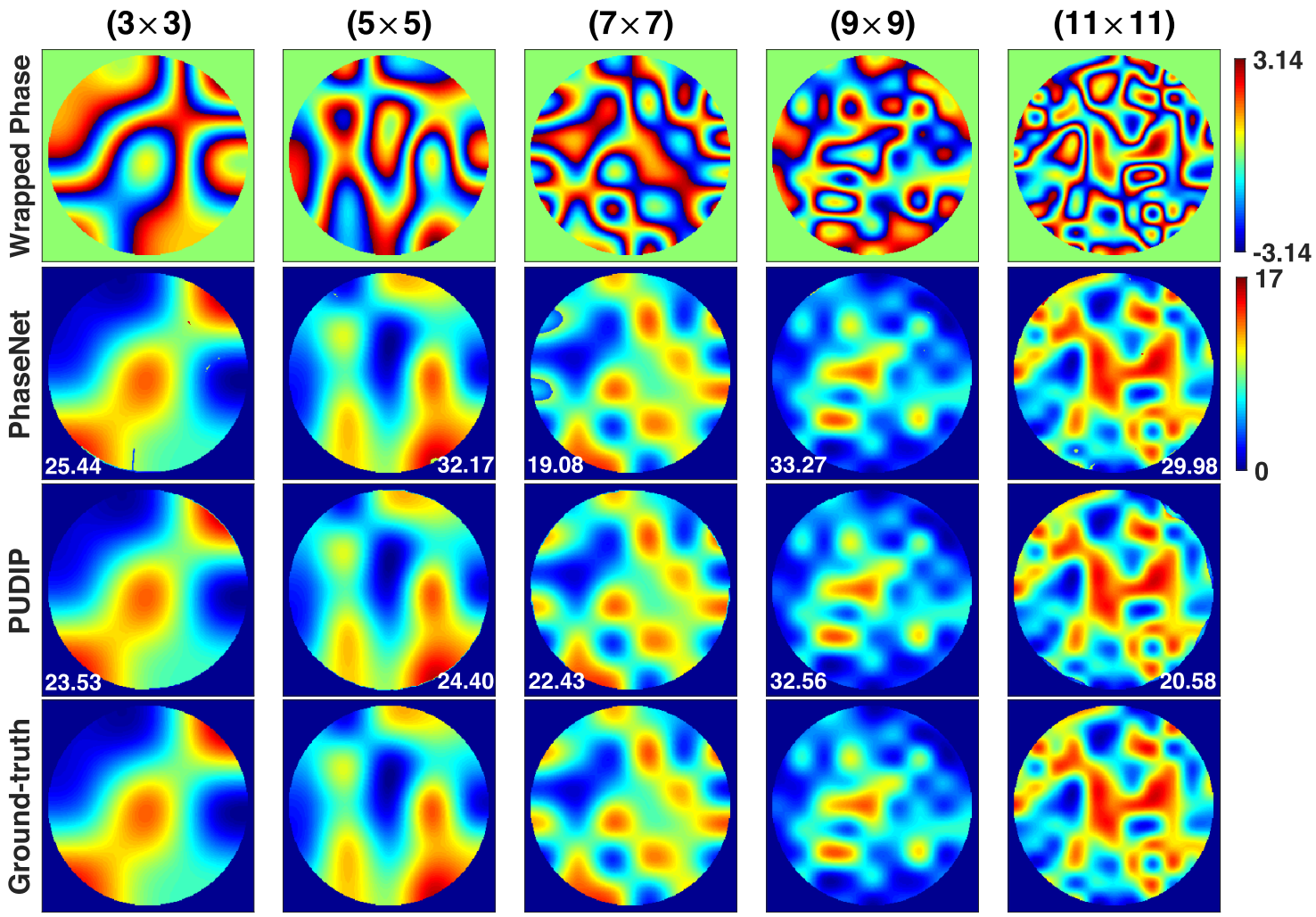

Fig. 8. Unwrapped-phase images of simulated samples with diverse random distributions. From top to bottom: wrapped phase, results obtained by PhaseNet and our approach (PUDIP). The ground-truth images are presented in the last row. The numbers give the corresponding RSNR [dB].

TABLE IV

RSNR [dB] AND SSIM OF THE RECONSTRUCTED-PHASE IMAGES Versus the Size of the Random Matrix. The Metrics Are Averaged Over Four SAMPles For EACH Size. For EACH SAmple, We RePeAted Five Times the ReCONSTRUCTIONS OF OUR METHOD. THE REPORTED RSNR AND SSIM OF PUDIP ARE THEN THE AVERAGE OF TWENTY EXPERIMENTS FOR EACH SIZE

\begin{tabular}{|c|c|c|c|c|c|c|c|}
\hline & Matrix size & GA & LS & IRTV & PUMA & PhaseNet & PUDIP \\
\hline \multirow{5}{*}{ 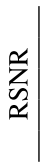 } & $(3 \times 3)$ & 4.18 & 3.84 & 3.60 & 3.72 & 36.30 & 21.94 \\
\hline & $(5 \times 5)$ & 5.57 & 5.55 & 4.87 & 5.39 & 31.89 & 21.51 \\
\hline & $(7 \times 7)$ & 5.32 & 6.28 & 5.72 & 5.41 & 21.97 & 19.98 \\
\hline & $(9 \times 9)$ & 5.53 & 6.19 & 5.47 & 5.99 & 39.71 & 20.80 \\
\hline & $(11 \times 11)$ & 5.71 & 6.88 & 6.82 & 6.88 & 23.63 & 18.65 \\
\hline \multirow{5}{*}{$\underset{\Sigma}{\Sigma}$} & $(3 \times 3)$ & 0.7361 & 0.7222 & 0.7253 & 0.7065 & 0.9920 & 0.9699 \\
\hline & $(5 \times 5)$ & 0.6828 & 0.6506 & 0.6592 & 0.6478 & 0.9567 & 0.9588 \\
\hline & $(7 \times 7)$ & 0.6636 & 0.6495 & 0.6348 & 0.6403 & 0.9576 & 0.9530 \\
\hline & $(9 \times 9)$ & 0.6511 & 0.7020 & 0.6579 & 0.6872 & 0.9637 & 0.9294 \\
\hline & $(11 \times 11)$ & 0.6532 & 0.6481 & 0.6574 & 0.6557 & 0.9234 & 0.9344 \\
\hline
\end{tabular}

a multiple of $2 \pi$. The SSIM metric is less sensitive to isolated erroneous cases and the discrepancy is much smaller. However, for some samples, PhaseNet wrongly estimates the phase over large areas inside the object (third column of Figure 8). Our method is more stable in its ability to unwrap the phase due to its feedback mechanism.

Let us observe that the results of PUDIP are still imperfect, in the sense that a few pixels of the output deviate from the ground-truth. However, these are inconspicuous. Based on our experiments, it appears that the results of PUDIP are generally superior to those of the other methods when the conditions are
TABLE V

RSNR [dB] AND SSIM OF THE RECONSTRUCTED-PHASE IMAGES VeRSUS THE NOISE LEVEL. THE RSNR AND SSIM OF OUR METHOD (PUDIP) ARE THE AVERAGE OF FIVE EXPERIMENTS

\begin{tabular}{|c|c|c|c|c|c|c|c|}
\hline & Noise level (dB) & GA & LS & IRTV & PUMA & PhaseNet & PUDIP \\
\hline \multirow{3}{*}{ 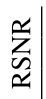 } & 22.80 & -3.58 & 1.67 & 2.32 & 2.34 & 3.24 & 20.51 \\
\hline & & -3.21 & 2.01 & 2.8 & 2. & 0.9 & 20.94 \\
\hline & 11.82 & 2.81 & 2.36 & 3.13 & 3.13 & 2.45 & 20.80 \\
\hline \multirow{3}{*}{$\begin{array}{l}\sum_{n} \\
\text { n }\end{array}$} & & 0.0072 & 0.1488 & 0.1619 & & & 0.9895 \\
\hline & 15.70 & 0.0081 & 0.1260 & 0.1493 & 0.1502 & -0.1402 & 0.9913 \\
\hline & 11.82 & 0.0023 & 0.1022 & 0.1195 & 0.1318 & 0.2046 & 0.9905 \\
\hline
\end{tabular}

difficult, and otherwise equivalent, which should make PUDIP of interest for practitioners. Note that when the task of phase unwrapping is extremely difficult, there are few failure cases. However, the failed results obtained by our approach are not worse than other methods. We provide all the results in the supplementary materials.

\section{Phase Unwrapping in Presence of Structured Noise}

In digital holography microscopy, the noise is mainly characterized by speckle noise [73] that corrupts the image before the wrapping operation. To assess the robustness of our method, we perturbed the (unwrapped) first kind of sample (cropping angle $135^{\circ}$ ) with speckle noise [74]. We added three levels of noise $\{11.8,15.7,22.8\} \mathrm{dB}$ (Figure 9) and computed the metrics with respect to the perturbed images.

The performances of the baseline methods are affected by the structured noise and fail to correctly unwrap the images (Table V). Note that their poor performance mainly 

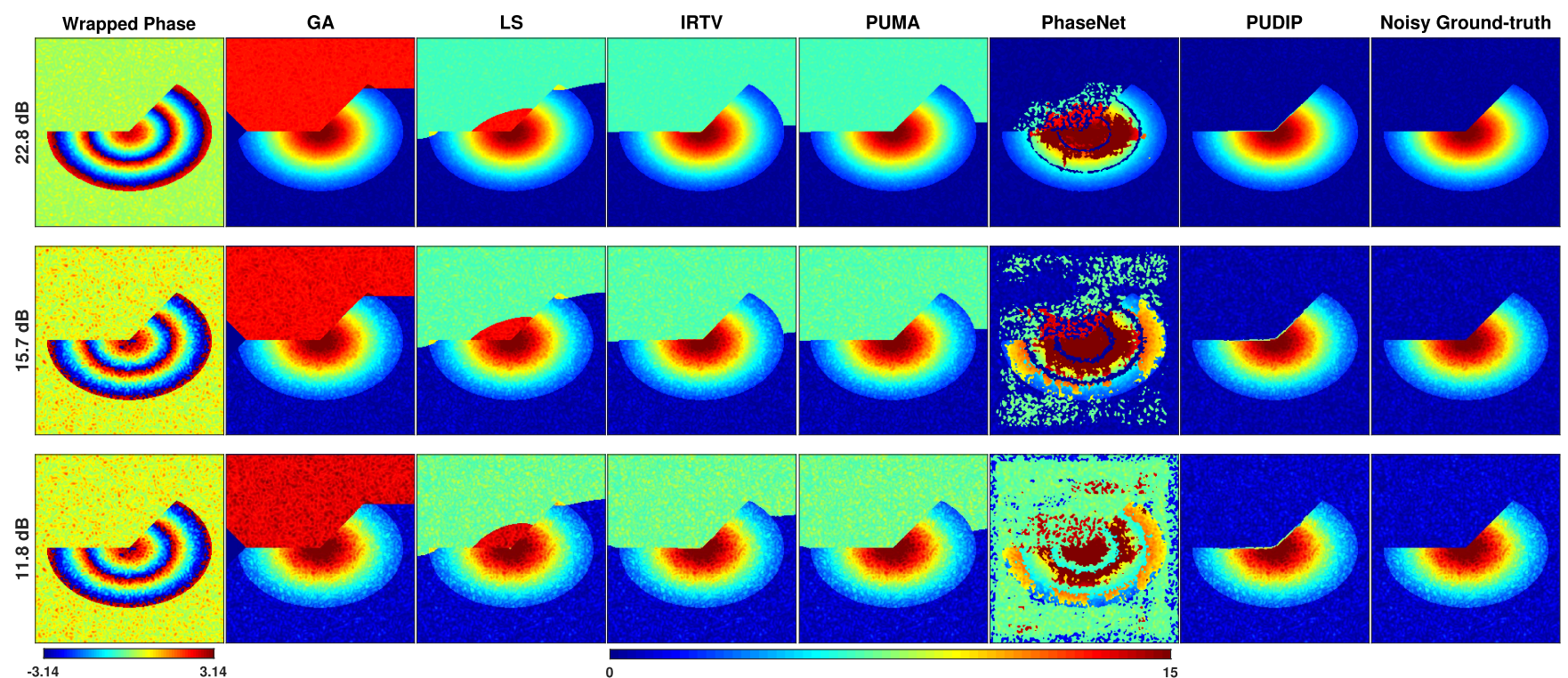

Fig. 9. Reconstructed unwrapped-phase images of simulated samples with diverse speckle noise. From left to right: wrapped phase, results obtained by GA, LS, IRTV, PUMA, PhaseNet, and our approach (PUDIP). The noisy ground-truth images are presented in the last column.

comes from the blocky errors mentioned in the previous experiments of Section V-C. The noise exacerbates the difficulty to recover the edges of the cropped ellipses. In the presence of noise, the performance of PhaseNet collapses, which is expected as this supervised method was trained on a noiseless dataset. PUDIP is stable, in that it correctly unwraps the phase, at the possible exception of few pixels at the border. It is worthy to mention that the robustness to noise is different from denoising, since we do not target at reducing the noise during the unwrapping process. This happens to other methods as well. When unwrapping is successful, one can then denoise the recovered phase image with any state-of-the-art denoising algorithms.

\section{CONCLUSION}

We proposed a general iterative framework PUDIP that takes advantage of model-based approaches and deep priors for two-dimensional phase unwrapping. The iterative inversion algorithm is based on a forward model that ensures consistency with the measurements and a generative network that learns the implicit knowledge of the image automatically. Further, the prior generated by the convolutional neural network without ground-truth overcomes the limitation of conventional supervised-learning strategies which need large-scale or tailored training datasets. We have validated our approach on simulated data with diverse challenging settings in which the unwrapped phase has many discontinuities. Numerical experiments have shown that the proposed method outperforms state-of-the-art conventional or network-based methods in many configurations. In addition, we have also applied our framework to single and time-lapse measurements of organoids, which are particularly large and complex samples. PUDIP can help in all instances of optical imaging that acquire wrapped phase data, quantitative phase imaging as well as more sophisticated tomographic schemes [75]. We believe that PUDIP should be of interest to practitioners. The substantial improvement of our method and the quality of reconstruction effectively allow the application of quantitative phase imaging to thick and complex three-dimensional samples, from which subsequent image processing can be carried on with higher reliability.

\section{ACKNOWLEDGMENT}

The authors would like to thank the China Scholarship Council for supporting the visit of the first co-author.

\section{REFERENCES}

[1] A. C. Rios and H. Clevers, "Imaging organoids: A bright future ahead," Nature Methods, vol. 15, no. 1, pp. 24-26, 2018.

[2] G. Rossi, A. Manfrin, and M. P. Lutolf, "Progress and potential in organoid research," Nature Rev. Genet., vol. 19, no. 11, pp. 671-687, Nov. 2018.

[3] M. Mir, B. Bhaduri, R. Wang, R. Zhu, and G. Popescu, "Quantitative phase imaging," in Progress in Optics, vol. 57. Amsterdam, The Netherlands: Elsevier, 2012, pp. 133-217.

[4] Y. Park, G. Popescu, K. Badizadegan, R. R. Dasari, and M. S. Feld, "Diffraction phase and fluorescence microscopy," Opt. Exp., vol. 14, no. 18, pp. 8263-8268, 2006.

[5] N. Pavillon et al., "Cell morphology and intracellular ionic homeostasis explored with a multimodal approach combining epifluorescence and digital holographic microscopy," J. Biophoton., vol. 3, no. 7, pp. 432-436, Mar. 2010.

[6] X. Quan, K. Nitta, O. Matoba, P. Xia, and Y. Awatsuji, "Phase and fluorescence imaging by combination of digital holographic microscopy and fluorescence microscopy," Opt. Rev., vol. 22, no. 2, pp. 349-353, Apr. 2015.

[7] S. Chowdhury, W. J. Eldridge, A. Wax, and J. A. Izatt, "Spatial frequency-domain multiplexed microscopy for simultaneous, singlecamera, one-shot, fluorescent, and quantitative-phase imaging," Opt. Lett., vol. 40, no. 21, pp. 4839-4842, 2015.

[8] Y. Park, C. Depeursinge, and G. Popescu, "Quantitative phase imaging in biomedicine," Nature Photon., vol. 12, no. 10, pp. 578-589, 2018.

[9] C. Hu and G. Popescu, Quantitative Phase Imaging: Principles and Applications. Cham, Switzerland: Springer, 2019, pp. 1-24.

[10] D. C. Ghiglia and M. D. Pritt, Two-Dimensional Phase Unwrapping: Theory, Algorithms, and Software, vol. 4. New York, NY, USA: Wiley, 1998.

[11] R. M. Goldstein, H. A. Zebker, and C. L. Werner, "Satellite radar interferometry: Two-dimensional phase unwrapping," Radio Sci., vol. 23, no. 4, pp. 713-720, Aug. 1988

[12] X. Su and W. Chen, "Reliability-guided phase unwrapping algorithm: A review," Opt. Lasers Eng., vol. 42, no. 3, pp. 245-261, Sep. 2004. 
[13] D. C. Ghiglia and L. A. Romero, "Minimum $L^{p}$-norm two-dimensional phase unwrapping," J. Opt. Soc. Amer. A, Opt. Image Sci., vol. 13, no. 10, pp. 1999-2013, 1996.

[14] D. C. Ghiglia and L. A. Romero, "Robust two-dimensional weighted and unweighted phase unwrapping that uses fast transforms and iterative methods," J. Opt. Soc. Amer. A, Opt. Image Sci., vol. 11, no. 1, pp. 107-117, 1994.

[15] W. He, L. Xia, and F. Liu, "Sparse-representation-based direct minimum $L^{p}$-norm algorithm for MRI phase unwrapping," Comput. Math. Methods Med., vol. 2014, Mar. 2014, Art. no. 134058.

[16] G. Nico, G. Palubinskas, and M. Datcu, "Bayesian approaches to phase unwrapping: Theoretical study," IEEE Trans. Signal Process., vol. 48, no. 9, pp. 2545-2556, Sep. 2000.

[17] L. Ying, Z.-P. Liang, D. C. Munson, Jr., R. Koetter, and B. J. Frey, "Unwrapping of MR phase images using a Markov random field model," IEEE Trans. Med. Imag., vol. 25, no. 1, pp. 128-136, Jan. 2006.

[18] Z.-P. Liang, "A model-based method for phase unwrapping," IEEE Trans. Med. Imag., vol. 15, no. 6, pp. 893-897, Dec. 1996.

[19] H. Takajo and T. Takahashi, "Least-squares phase estimation from the phase difference," J. Opt. Soc. Amer. A, Opt. Image Sci., vol. 5, no. 3, pp. 416-425, 1988.

[20] J. M. Bioucas-Dias and G. Valadao, "Phase unwrapping via graph cuts," IEEE Trans. Image Process., vol. 16, no. 3, pp. 698-709, Mar. 2007.

[21] B. Zhang, L. Wei, S. Li, and Q. Hu, "Precise Markov random field model-based phase unwrapping method for airborne interferometric synthetic aperture radar imaging," J. Appl. Remote Sens., vol. 12, no. 3, 2018, Art. no. 035019.

[22] L. Zhou, D. Chai, Y. Xia, and C. Xie, "An extended PUMA algorithm for multibaseline InSAR DEM reconstruction,” Int. J. Remote Sens., vol. 40, no. 20, pp. 7830-7851, 2019.

[23] L. Condat, D. Kitahara, and A. Hirabayashi, "A convex lifting approach to image phase unwrapping," in Proc. IEEE Int. Conf. Acoust., Speech Signal Process. (ICASSP), May 2019, pp. 1852-1856.

[24] U. S. Kamilov, I. N. Papadopoulos, M. H. Shoreh, D. Psaltis, and M. Unser, "Isotropic inverse-problem approach for two-dimensional phase unwrapping," J. Opt. Soc. Amer. A, Opt. Image Sci., vol. 32, no. 6, pp. 1092-1100, 2015.

[25] S. Lefkimmiatis, J. P. Ward, and M. Unser, "Hessian schatten-norm regularization for linear inverse problems," IEEE Trans. Image Process., vol. 22, no. 5, pp. 1873-1888, May 2013.

[26] S. Boyd, N. Parikh, E. Chu, B. Peleato, and J. Eckstein, "Distributed optimization and statistical learning via the alternating direction method of multipliers," Found. Trends Mach. Learn., vol. 3, no. 1, pp. 1-122, Jan. 2011.

[27] J. Besag, "On the statistical analysis of dirty pictures," J. Roy. Stat. Soc. B, Methodol., vol. 48, no. 3, pp. 259-279, 1986.

[28] K. Itoh, "Analysis of the phase unwrapping algorithm," Appl. Opt., vol. 21, no. 14 , p. $2470,1982$.

[29] B. Bhaduri, H. Pham, M. Mir, and G. Popescu, "Diffraction phase microscopy with white light," Opt. Lett., vol. 37, no. 6, pp. 1094-1096, 2012.

[30] Z. Wang et al., "Spatial light interference microscopy (SLIM)," Opt. Exp., vol. 19, no. 2, pp. 1016-1026, 2011.

[31] C. J. Mann, P. R. Bingham, V. C. Paquit, and K. W. Tobin, "Quantitative phase imaging by three-wavelength digital holography," Opt. Exp., vol. 16, no. 13, pp. 9753-9764, 2008.

[32] Y. Li, W. Xiao, and F. Pan, "Multiple-wavelength-scanning-based phase unwrapping method for digital holographic microscopy," Appl. Opt., vol. 53, no. 5, pp. 979-987, 2014.

[33] C. Hu and G. Popescu, "Quantitative phase imaging (QPI) in neuroscience," IEEE J. Sel. Topics Quantum Electron., vol. 25, no. 1, pp. 1-9, Jan. 2019.

[34] B. Zhu, J. Z. Liu, S. F. Cauley, B. R. Rosen, and M. S. Rosen, "Image reconstruction by domain-transform manifold learning," Nature, vol. 555, no. 7697, pp. 487-492, 2018

[35] M. T. McCann, K. H. Jin, and M. Unser, "Convolutional neural networks for inverse problems in imaging: A review," IEEE Signal Process. Mag., vol. 34, no. 6, pp. 85-95, Nov. 2017.

[36] H. Wang et al., "Deep learning enables cross-modality superresolution in fluorescence microscopy," Nature Methods, vol. 16, no. 1, pp. 103-110, Jan. 2019.

[37] K. H. Jin, M. T. McCann, E. Froustey, and M. Unser, "Deep convolutional neural network for inverse problems in imaging," IEEE Trans. Image Process., vol. 26, no. 9, pp. 4509-4522, Sep. 2017.

[38] C. Yan, B. Gong, Y. Wei, and Y. Gao, "Deep multi-view enhancement hashing for image retrieval," IEEE Trans. Pattern Anal. Mach. Intell., vol. 43, no. 4, pp. 1445-1451, Apr. 2021.
[39] C. Yan, B. Shao, H. Zhao, R. Ning, Y. Zhang, and F. Xu, "3D room layout estimation from a single RGB image," IEEE Trans. Multimedia, vol. 22, no. 11, pp. 3014-3024, Nov. 2020.

[40] C. Yan, Z. Li, Y. Zhang, Y. Liu, X. Ji, and Y. Zhang, "Depth image denoising using nuclear norm and learning graph model," ACM Trans. Multimedia Comput., Commun., Appl., vol. 16, no. 4, pp. 1-17, Jan. 2021.

[41] Y. Jo et al., "Quantitative phase imaging and artificial intelligence: A review," IEEE J. Sel. Topics Quantum Electron., vol. 25, no. 1, Jan./Feb. 2019, Art. no. 6800914.

[42] G. Barbastathis, A. Ozcan, and G. Situ, "On the use of deep learning for computational imaging," Optica, vol. 6, no. 8, pp. 921-943, 2019.

[43] W. Schwartzkopf, T. E. Milner, J. Ghosh, B. L. Evans, and A. C. Bovik, "Two-dimensional phase unwrapping using neural networks," in Proc. 4th IEEE Southwest Symp. Image Anal. Interpretation, Austin, TX, USA, Apr. 2000, pp. 274-277.

[44] K. He, X. Zhang, S. Ren, and J. Sun, "Deep residual learning for image recognition," in Proc. IEEE Conf. Comput. Vis. Pattern Recognit. (CVPR), Las Vegas, NV, USA, Jun. 2016, pp. 770-778.

[45] G. Dardikman and N. T. Shaked, "Phase unwrapping using residual neural networks," in Computational Optical Sensing and Imaging. Orlando, FL, USA: Optical Society of America, Jun. 2018, Paper CW3B.5.

[46] G. E. Spoorthi, S. Gorthi, and R. K. S. S. Gorthi, "PhaseNet: A deep convolutional neural network for two-dimensional phase unwrapping," IEEE Signal Process. Lett., vol. 26, no. 1, pp. 54-58, Jan. 2019.

[47] T. Zhang et al., "Rapid and robust two-dimensional phase unwrapping via deep learning," Opt. Exp., vol. 27, no. 16, pp. 23173-23185, 2019.

[48] K. Wang, Y. Li, Q. Kemao, J. Di, and J. Zhao, "One-step robust deep learning phase unwrapping," Opt. Exp., vol. 27, no. 10, pp. 15100-15115, 2019.

[49] J. Zhang, X. Tian, J. Shao, H. Luo, and R. Liang, "Phase unwrapping in optical metrology via denoised and convolutional segmentation networks," Opt. Exp., vol. 27, no. 10, pp. 14903-14912, 2019.

[50] C. Li, Y. Tian, and J. Tian, "A method for single image phase unwrapping based on generative adversarial networks," in Proc. 11th Int. Conf. Digit. Image Process. (ICDIP), Guangzhou, China, Aug. 2019, pp. 272-278.

[51] G. Dardikman-Yoffe, D. Roitshtain, S. K. Mirsky, N. A. Turko, M. Habaza, and N. T. Shaked, "PhUn-Net: Ready-to-use neural network for unwrapping quantitative phase images of biological cells," Biomed. Opt. Exp., vol. 11, no. 2, pp. 1107-1121, Feb. 2020.

[52] J. H. R. Chang, C.-L. Li, B. Poczos, B. V. K. V. Kumar, and A. C. Sankaranarayanan, "One network to solve them all-solving linear inverse problems using deep projection models," in Proc. IEEE Int. Conf. Comput. Vis. (ICCV), Venice, Italy, Oct. 2017, pp. 5888-5897.

[53] H. Gupta, K. H. Jin, H. Q. Nguyen, M. T. McCann, and M. Unser, "CNN-based projected gradient descent for consistent CT image reconstruction," IEEE Trans. Med. Imag., vol. 37, no. 6, pp. 1440-1453, Jun. 2018

[54] F. Yang, T.-A. Pham, H. Gupta, M. Unser, and J. Ma, "Deep-learning projector for optical diffraction tomography," Opt. Exp., vol. 28, no. 3, pp. 3905-3921, 2020

[55] V. Lempitsky, A. Vedaldi, and D. Ulyanov, "Deep image prior," in Proc. IEEE/CVF Conf. Comput. Vis. Pattern Recognit., Salt Lake City, UT, USA, Jun. 2018, pp. 9446-9454.

[56] K. Gong, C. Catana, J. Qi, and Q. Li, "PET image reconstruction using deep image prior," IEEE Trans. Med. Imag., vol. 38, no. 7, pp. 1655-1665, Jul. 2019.

[57] L. Condat, "Discrete total variation: New definition and minimization," SIAM J. Imag. Sci., vol. 10, no. 3, pp. 1258-1290, 2017.

[58] M. D. Pritt, "Congruence in least-squares phase unwrapping," in Proc. IEEE Int. Geosci. Remote Sens. Symp. Remote Sens., Sci. Vis. Sustain. Develop. (IGARSS), Singapore, Aug. 1997, pp. 875-877.

[59] K. C. Zhou and R. Horstmeyer, "Diffraction tomography with a deep image prior," Opt. Exp., vol. 28, no. 9, pp. 12872-12896, 2020.

[60] E. Bostan, R. Heckel, M. Chen, M. Kellman, and L. Waller, "Deep phase decoder: Self-calibrating phase microscopy with an untrained deep neural network," Optica, vol. 7, no. 6, pp. 559-562, 2020.

[61] F. Wang et al., "Phase imaging with an untrained neural network," Light: Sci. Appl., vol. 9, no. 1, pp. 1-7, Dec. 2020

[62] O. Ronneberger, P. Fischer, and T. Brox, "U-Net: Convolutional networks for biomedical image segmentation," in Proc. Int. Conf. Med. Image Comput. Comput.-Assist. Intervent., Münich, Germany, Oct. 2015, pp. 234-241.

[63] K. He, X. Zhang, S. Ren, and J. Sun, "Delving deep into rectifiers: Surpassing human-level performance on ImageNet classification," in Proc. IEEE Int. Conf. Comput. Vis. (ICCV), Santiago, Chile, Dec. 2015, pp. 1026-1034. 
[64] T. Vicar et al., "Cell segmentation methods for label-free contrast microscopy: Review and comprehensive comparison," BMC Bioinf., vol. 20, no. 1, p. 360, Dec. 2019.

[65] D. P. Kingma and J. Ba, "Adam: A method for stochastic optimization," in Proc. 3rd Int. Conf. Learn. Represent. (ICLR), San Diego, CA, USA, May 2015, pp. 1-15.

[66] N. Ketkar, "Introduction to Pytorch," in Deep Learning With PyThon. Berkeley, CA, USA: Springer, 2017, pp. 195-208.

[67] N. Brandenberg et al., "High-throughput automated organoid culture via stem-cell aggregation in microcavity arrays," Nature Biomed. Eng., vol. 4, pp. 863-874, Jun. 2020

[68] X. Li and M. T. Orchard, "New edge-directed interpolation," IEEE Trans. Image Process., vol. 10, no. 10, pp. 1521-1527, Oct. 2001.

[69] X. Yin, B. E. Mead, H. Safaee, R. Langer, J. M. Karp, and O. Levy, "Engineering stem cell organoids," Cell Stem Cell, vol. 18, no. 1, pp. 25-38, Jan. 2016.

[70] A. C. Kak, M. Slaney, and G. Wang, "Principles of computerized tomographic imaging," Med. Phys., vol. 29, no. 1, p. 107, 2002.

[71] K. L. Fair, J. Colquhoun, and N. R. F. Hannan, "Intestinal organoids for modelling intestinal development and disease," Phil. Trans. Roy. Soc. B, Biol. Sci., vol. 373, no. 1750, Jul. 2018, Art. no. 20170217.

[72] M. Feit and J. Fleck, "Beam nonparaxiality, filament formation, and beam breakup in the self-focusing of optical beams," J. Opt. Soc. Amer. B, Opt. Phys., vol. 5, no. 3, pp. 633-640, 1988.

[73] V. Bianco et al., "Strategies for reducing speckle noise in digital holography," Light: Sci. Appl., vol. 7, no. 1, pp. 1-16, Dec. 2018.

[74] J. W. Goodman, Speckle Phenomena in Optics: Theory and Applications. Greenwood Village, CO, USA: Roberts and Company Publishers, 2007.

[75] D. Jin, R. Zhou, Z. Yaqoob, and P. T. So, "Tomographic phase microscopy: Principles and applications in bioimaging [Invited]," J. Opt. Soc. Amer. B, Opt. Phys., vol. 34, no. 5, pp. B64-B77, 2017.

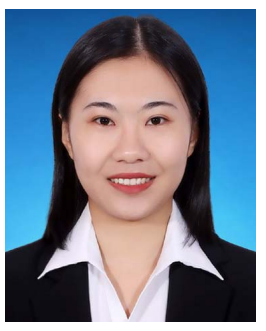

Fangshu Yang (Student Member, IEEE) received the B.S. degree in information and computing sciences from Shandong University of Science and Technology, Qingdao, China, in 2014, and the Ph.D. degree in applied mathematics from Harbin Institute of Technology, Harbin, China, in 2021.

She was a Visiting Ph.D. Student of the Biomedical Imaging Group, EPFL, Switzerland, from 2018 to 2020. Her main research interests include deep learning, image processing, and inverse problems, mainly in optical imaging and seismic exploration.

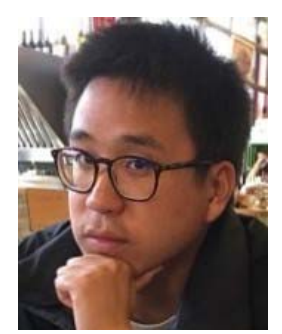

Thanh-An Pham (Graduate Student Member, IEEE) received the M.S. degree in bioengineering from the École Polytechnique Fédérale de Lausanne (EPFL), Lausanne, Switzerland, in 2016, where he is currently pursuing the Ph.D. degree in electrical engineering.

His main research interests include inverse problems for computational imaging, notably in optical diffraction tomography and single-molecule localization microscopy.

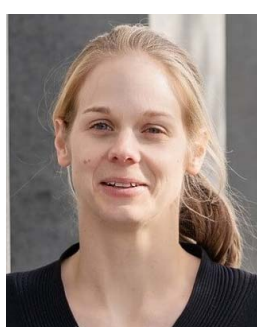

Nathalie Brandenberg received the Ph.D. degree in bioengineering from the Laboratory of Prof. Matthias Lütolf, EPFL. She was trained as a Bioengineer at EPFL. She co-founded and became the Co-CEO of SUN bioscience, an EPFL spin-off active in the industrial production of stem cell cultures for precision medicine. Her work has led to several publications, some of which in high impact journals, and several patents. Her research focuses on developing scalable stem-cell derived organoids models, namely miniature tissues in a dish using cutting-edge engineering technologies to enable precision medicine for both the clinics and pharmaceutical pre- and post-clinical developments with a current focus on cystic fibrosis and oncology.

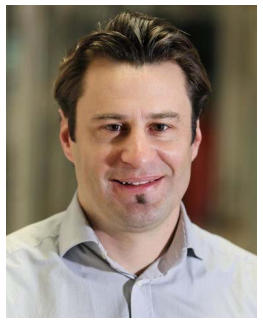

Matthias P. Lütolf received the Ph.D. degree from ETH Zurich (with Jeffrey Hubbell). He was trained as a Materials Engineer at ETH Zurich. He continued his research training as a Postdoctoral Fellow in stem cell biology (with Helen Blau) at Stanford University. He has served as the Director for the Institute of Bioengineering from 2014 to 2018 . He is currently a Full Professor at the EPFL's Institute of Bioengineering, with a cross appointment at the Institute of Chemical Sciences and Engineering. $\mathrm{He}$ is also an internationally recognized leader in the fields of stem cell bioengineering and tissue engineering. His research program uniquely combines stem cell biology with engineering principles and quantitative thinking. His team, composed of engineers, chemists, physicists, cell and developmental biologists, strives to develop technologies that have true biological and medicinal function and applicability. His work has led to more than 110 peer-reviewed scientific publications, many of which published in highly reputed journals, more than 25 patents, and the commercialization of several products. His current research in the his lab is focused on the bioengineering of miniature tissues, termed organoids, that are generated from self-organizing stem cells. He has received the ETH Medal for his Ph.D. degree.

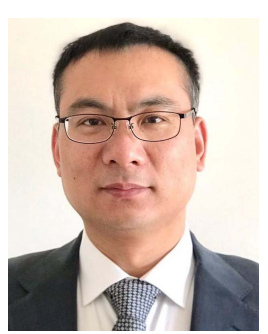

Jianwei Ma (Senior Member, IEEE) received the $\mathrm{Ph} . \mathrm{D}$. degree in engineering from Tsinghua University, Beijing, China, in 2002.

From 2006 to 2010, he was an Assistant Professor and an Associate Professor with the School of Aerospace, Tsinghua University. From 2010 to 2011, he was a Scientist with Florida State University. He has been a Postdoctoral Researcher and has a visiting experiences with the University of Cambridge, the University of Grenoble, the University of Göttingen, the Ecole des Mines de Paris, The University of Texas at Austin, and the University of California at Los Angeles. After 2011, he is a Professor with the School of Mathematics and the Vice Dean of the Institute of Artificial Intelligence, Harbin Institute of Technology, Harbin, China. He has been a Professor with the School of Earth and Space Sciences, Peking University, China, since 2020. His main research interests include sparse transforms, geophysical data processing, compressed sensing, inverse problems, and deep learning. He was a recipient of the NFSC Distinguished Young Scholars in 2016 and the Principal Investigator of the National Key Research and Development Program of China.

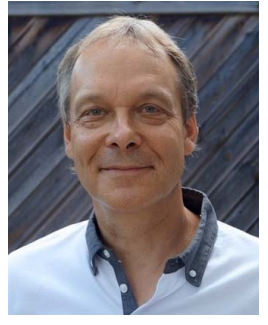

bioimaging.

Dr. Unser is an EURASIP Fellow in 2009 and a member of the Swiss Academy of Engineering Sciences. From 1985 to 1997, he was with the Biomedical Engineering and Instrumentation Program, National Institutes of Health, Bethesda, USA, conducting research on bioimaging. He was a recipient of several international prizes, including five IEEE-SPS Best Paper Awards, two Technical Achievement Awards from the IEEE (2008 SPS and EMBS 2010), the Technical Achievement Award from EURASIP in 2018, and the recent Career Achievement Award (IEEE EMBS 2020). He is the Founding Chair of the Technical Committee on Bio Imaging and Signal Processing (BISP) of the IEEE Signal Processing Society. He has served on the Editorial Board for most of the primary journals in his field, including the IEEE TRANSACTIONS ON MEDICAL IMAGING (an associate editor-inchief from 2003 to 2005), IEEE TRANS ACTIONS ON IMAGE PROCESSING, ProceEdings of THE IEEE, and SIAM Journal on Imaging Sciences. 